\title{
Site-Specific and Trigger-Activated Modification of Proteins by Means of Catalytic Hemin/G-quadruplex DNAzyme Nanostructures
}

\author{
Jordi F. Keijzer and Bauke Albada*
}

Cite This: https://dx.doi.org/10.1021/acs.bioconjchem.0c00422

Read Online

ACCESS

Wlll Metrics \& More

Article Recommendations

Supporting Information

ABSTRACT: Catalytic nanostructures have the potency to mimic enzymatic features. In this paper, we show that the complex between hemin and G-quadruplex DNA efficiently catalyzes the modification of proteins with $N$-methyl luminol derivatives. Final conversions are reached within 15-30 min, and LC-MS analysis of tryptic digests of the proteins shows that the reaction proceeds with chemoselectivity for electron-rich aromatic residues ( $\mathrm{Tyr} \gg$ Trp), and the site-specificity of the modification depends on the sequence and secondary structure folding of the G-quadruplex nanostructure. Furthermore, the modification can be applied on proteins with different biomedical functions, and the nanostructure can be designed to contain a regulatory element in order to regulate protein modification by an external stimulus.

$\mathrm{T}$ he hemin/G-quadruplex (hGQ) nanostructure is a DNAbased catalyst that can mimic reactions of peroxidase enzymes. $^{1-3}$ The activity of these so-called hGQDNAzymes is determined by the sequence and secondary structure formed by the layers of guanine tetrads. ${ }^{4,5}$ Further enhancement of the catalytic activity $\left(k_{\text {cat }}\right)$ can be achieved by nucleotide supplements, ${ }^{6}$ or by conjugation of the hGQDNAzyme to an aptamer sequence that binds to the substrate. ${ }^{7,8}$ Such so-called nucleoapzymes can be subjected to rational design ${ }^{9}$ or incorporation into supramolecular assemblies. ${ }^{8}$ The predictable formation of the catalytically active DNAzyme nanostructure has resulted in its incorporation into complex oligonucleotide assemblies of which the designed activity depends on an external trigger. ${ }^{10}$ Apart from the oxidation of chemical substrates in sensor-type setups, ${ }^{10}$ some conversions mimic biological processes such as the oxidation of dopamine to aminochrome or of $\mathrm{N}$-hydroxy-L-arginine to nitric oxide and L-citrulline. ${ }^{7} \mathrm{We}$ now establish hGQ DNAzymes as potent catalysts for sitespecific oxidative protein modification.

Protein modification is ideally performed in a rapid, efficient, and site-specific manner. ${ }^{11,12}$ The latest methods apply bioorthogonal click-chemistry reactions that are superior in rate and selectivity. ${ }^{13,14}$ Alternatively, synthetic catalysts ${ }^{15}$ or enzymes $^{16,17}$ have been applied for the modification of native proteins, or for the conversion of (a) genetically encoded handle(s). ${ }^{18-21}$ However, when it comes to the application of biomimetic catalytic species for protein modification, only a few methods exist such as the pyridoxal-5-phosphate method for the modification of the protein N-terminus, ${ }^{22}$ or the hemincatalyzed or $\mathrm{Ru}$ (bipy) ${ }_{3}$-induced modification of Tyr residues. ${ }^{23}$

In order to test if the hGQ DNAzyme nanostructure would allow us to design catalysts ${ }^{6}$ that displays variations in protein modification ability, and responds to external triggers, we used hGQ DNAzymes based on various topologies ${ }^{24}$ (Figure 1A) as

A

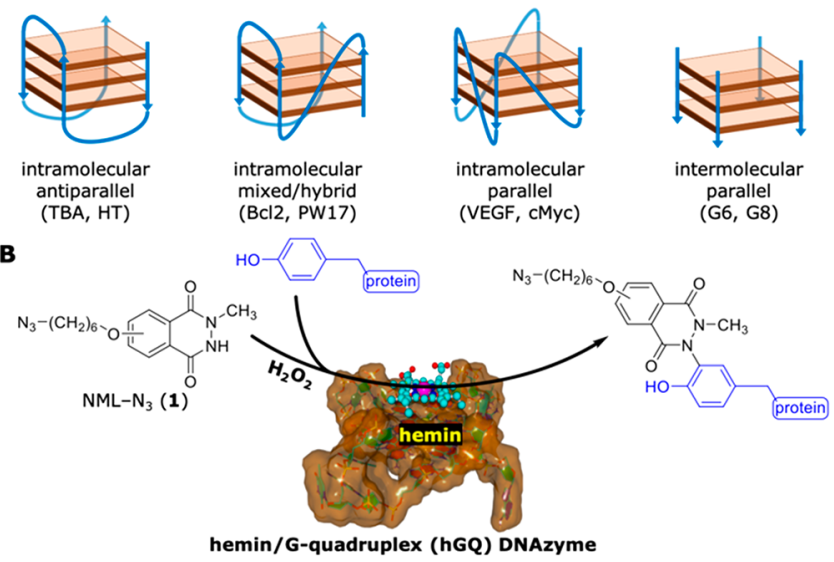

Figure 1. (A) G-Quadruplex topologies used to construct the DNAzymes in this study (for G6 and G8, only three G tetrads are shown). (B) Hemin/G-quadruplex (hGQ) DNAzyme catalyzed modification of a protein-based tyrosine residue with $\mathrm{N}$-methyl luminol derivative $\mathbf{1}$ in the presence of $\mathrm{H}_{2} \mathrm{O}_{2}$.

Received: July 24, 2020

Revised: August 21, 2020

Published: September 10, 2020 
Table 1. Details of the hGQDNAzyme Catalyzed Modification of Lysozyme or Thrombin with $\mathrm{N}$-Methyl Luminol Derivative 1

\begin{tabular}{|c|c|c|c|c|c|c|c|c|c|}
\hline \multirow[b]{2}{*}{ code } & \multirow[b]{2}{*}{ GQ type } & \multicolumn{4}{|c|}{ lysozyme modification $^{a}(\%)$} & \multicolumn{4}{|c|}{ thrombin modification $^{b}(\%)$} \\
\hline & & total & 1 & 2 & $>2$ & total & 1 & 2 & $>2$ \\
\hline \multirow[t]{2}{*}{$\mathrm{H}$} & hemin alone & 2 & 2 & - & - & 5 & 5 & - & - \\
\hline & & \multicolumn{4}{|c|}{ Tyr: 23} & \multicolumn{4}{|c|}{ Tyr: 85} \\
\hline G6 & intermol. parallel & 36 & 36 & - & - & 81 & 58 & 20 & 3 \\
\hline \multirow[t]{3}{*}{ G8 } & & 78 & 69 & 8 & 1 & 82 & 58 & 20 & 4 \\
\hline & & \multicolumn{4}{|c|}{ Tyr: $20 / 23 / 53$} & \multicolumn{4}{|c|}{ Tyr: 85/114/134/190 } \\
\hline & & \multicolumn{4}{|c|}{ Trp: 123} & \multicolumn{4}{|c|}{ Trp: $148 / 190$} \\
\hline TBA & intramol. antiparallel & 35 & 35 & - & - & 29 & 25 & 4 & - \\
\hline \multirow[t]{3}{*}{ HT } & & 32 & 32 & - & - & 44 & 32 & 2 & - \\
\hline & & \multirow{2}{*}{\multicolumn{4}{|c|}{ Tyr: $20 / 23$}} & \multicolumn{4}{|c|}{ Tyr: $85 / 134$} \\
\hline & & & & & & \multicolumn{4}{|c|}{ Trp: 148} \\
\hline $\mathrm{Bcl} 2$ & intramol. mixed/hybrid & 72 & 62 & 10 & - & 96 & 69 & 27 & - \\
\hline \multirow[t]{3}{*}{ PW17 } & & 74 & 65 & 8 & 1 & 97 & 41 & 39 & 17 \\
\hline & & \multicolumn{4}{|c|}{ Tyr: $20 / 23$} & \multicolumn{4}{|c|}{ Tyr: $85 / 114 / 190$} \\
\hline & & \multicolumn{4}{|c|}{ Trp: 62} & \multicolumn{4}{|c|}{ Trp: 148} \\
\hline EA2 & intramol. parallel & 91 & 70 & 18 & 3 & 96 & 41 & 40 & 15 \\
\hline \multirow[t]{3}{*}{$\mathrm{cMyc}$} & & 96 & 63 & 27 & 6 & 94 & 59 & 27 & 8 \\
\hline & & \multicolumn{4}{|c|}{ Tyr: $20 / 23 / 53$} & \multicolumn{4}{|c|}{ Tyr: $85 / 114 / 134 / 190$} \\
\hline & & \multicolumn{4}{|c|}{ Trp: $62 / 123$} & \multicolumn{4}{|c|}{ Trp: 148/190/27 (LC) } \\
\hline
\end{tabular}

${ }^{a}$ Conditions: $10 \mu \mathrm{M}$ hemin, $10 \mu \mathrm{M}$ DNA, $140 \mu \mathrm{M}$ lysozyme, $700 \mu \mathrm{M} \mathrm{NML}-\mathrm{N}_{3}$ (1), and $2800 \mu \mathrm{M} \mathrm{H}_{2} \mathrm{O}_{2}$ (reaction time: 30 min). ${ }^{b}$ Conditions: 10 $\mu \mathrm{M}$ hemin, $10 \mu \mathrm{M}$ DNA, $42 \mu \mathrm{M}$ thrombin, $300 \mu \mathrm{M}$ NML- $\mathrm{N}_{3}$ (1), and $300 \mu \mathrm{M} \mathrm{H}_{2} \mathrm{O}_{2}$ (reaction time: $30 \mathrm{~min}$ ).
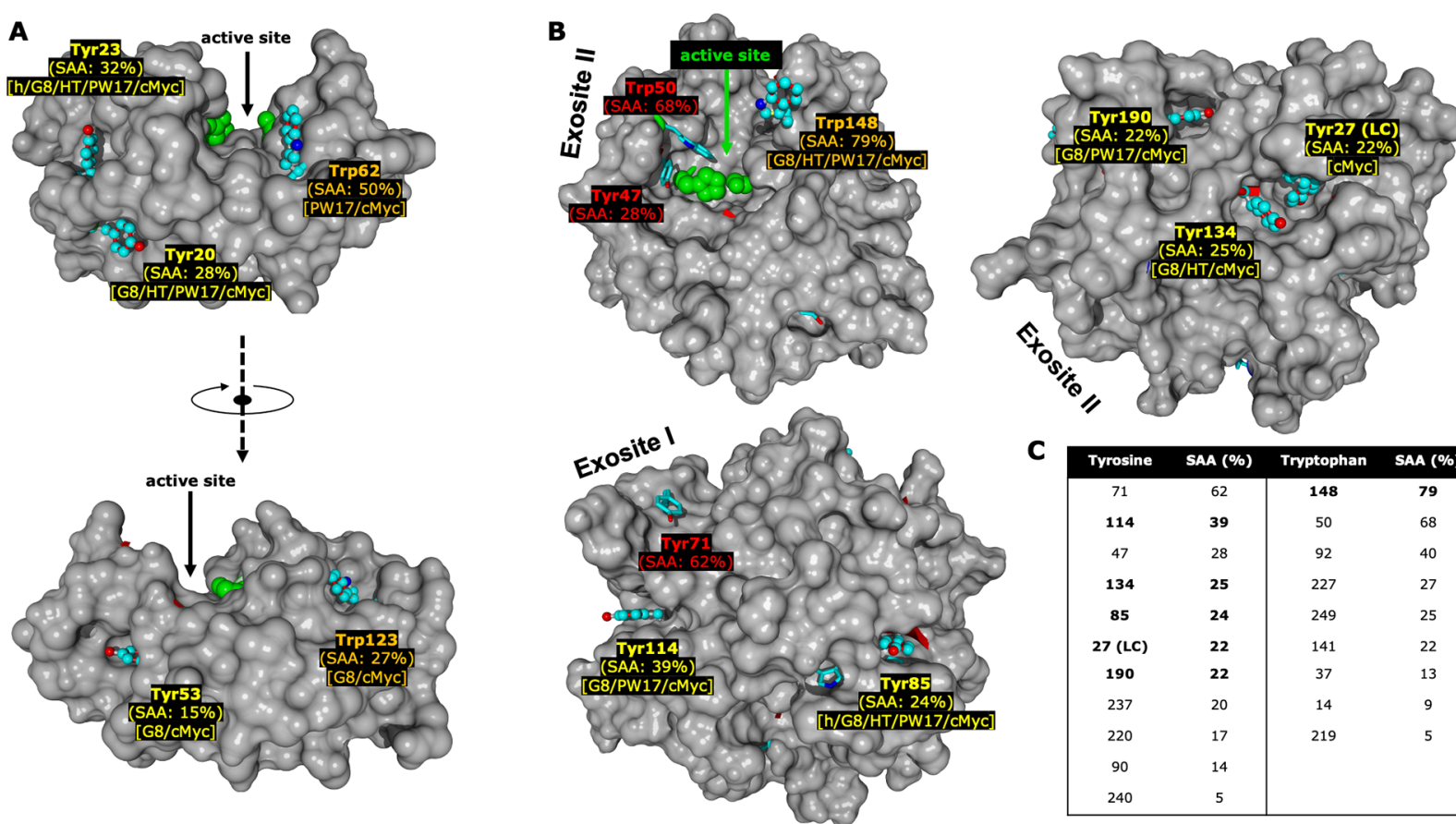

\begin{tabular}{|c|c|c|c|}
\hline Tyrosine & SAA (\%) & Tryptophan & SAA (\%) \\
\hline 71 & 62 & 148 & 79 \\
\hline 114 & 39 & 50 & 68 \\
\hline 47 & 28 & 92 & 40 \\
\hline 134 & 25 & 227 & 27 \\
\hline 85 & 24 & 249 & 25 \\
\hline 27 (LC) & 22 & 141 & 22 \\
\hline 190 & 22 & 37 & 13 \\
\hline 237 & 20 & 14 & 9 \\
\hline 220 & 17 & 219 & 5 \\
\hline 90 & 14 & & \\
\hline 240 & 5 & & \\
\hline
\end{tabular}

Figure 2. (A,B) Relevant sides of lysozyme (A) and human $\alpha$-thrombin (B) with the position and solvent accessible area (SAA) of the residues that were modified with the respective hGQDNAzymes (given between the square brackets; an " $h$ " indicates that this residue is also modified by hemin alone), or the Tyr/Trp residues in thrombin that have a high SAA but that are not modified (in red) [based on PDB-codes 3JIV (lysozyme) and 5EW2 (thrombin)]. Modified residues are displayed in ball-and-stick, unmodified residues as sticks; active site residues are shown in green ball display. (C) List of all Tyr and Trp residues in thrombin in decreasing SAA percentage, with the residues that are modified in bold (LC refers to the thrombin light chain; the other residues are on its heavy chain).

catalysts for the oxidative modification of proteins using $\mathrm{N}$ methyl luminol ${ }^{25,26}$ derivatives and hydrogen peroxide $\left(\mathrm{H}_{2} \mathrm{O}_{2}\right)$ (Figure 1B). Having the ability to design the local environment of a protein-modifying catalyst would allow closer mimicry of features that give enzymes the ability to perform highly selective protein modification. $^{23}$ To test this, we used lysozyme, thrombin, bovine serum albumin (BSA), and the therapeutically relevant immunoglobulin trastuzumab as a representative set that covers a large range of protein sizes, i.e., $14 \mathrm{kDa}$ for lysozyme to $150 \mathrm{kDa}$ for trastuzumab.

Lysozyme $(14.3 \mathrm{kDa})$ contains three tyrosine $(\mathrm{Tyr})$ residues with different solvent-accessible areas (SAA): Tyr23 (SAA: 32\%), Tyr20 (SAA: 28\%), and Tyr53 (SAA: 15\%). ${ }^{27}$ The presence of potentially competing aromatic amino acid residues 
tryptophan (Trp), phenylalanine, and histidine allowed assessment of the chemoselectivity of the reaction. Much to our delight, LC-MS analysis of the reaction mixtures revealed substantial levels (32-96\%) of lysozyme modification by various hGQ DNAzyme nanostructures in potassium-containing buffers (Table 1). Whereas only $2 \%$ of lysozyme modification was observed for hemin alone (Table 1) or unstructured ssDNA or dsDNA (see SI Table S2), the presence of G-quadruplex structures led to higher amounts of modified protein. Notable differences were observed for different Gquadruplex topologies: intramolecular parallel GQs formed the most active complexes, followed by the intramolecular mixed/ hybrid GQs and intermolecular parallel GQs, and with the intramolecular antiparallel GQs generating the least active hGQ DNAzymes. ${ }^{5}$ Similar trends have been observed for the oxidation of $\mathrm{ABTS}^{2-}$ by different hGQ structures. ${ }^{5}$ Furthermore, we also find that the most active sequences contain a $3^{\prime}$-end positioned A, which has been linked to enhanced catalytic activities of hGQDNAzymes. ${ }^{6,28}$ Therefore, we propose that the combination of steric bulk surrounding the catalytic center and the presence of appropriately positioned assisting nucleotides are causing the observed differences in activity. Time-resolved HPLC-analysis of the reaction mixtures revealed that the modification was nearly complete after $15 \mathrm{~min}$ (SI Figure S1).

Interestingly, conjugation of a lysozyme-binding aptamer (LBA) to G-quadruplex structures affected the ability of the hGQDNAzyme to modify the protein (SI Table S2). In general, higher $\mathrm{H}_{2} \mathrm{O}_{2}$ concentrations led to faster conversion, whereas higher NML and/or DNAzyme concentrations increased the number of modifications (SI Tables S3-S4). Furthermore, hGQDNAzyme-induced modifications resulted in a decrease in the glycanhydrolase activity (SI Table S5). ${ }^{29}$

The effect of G-quadruplex topology on the residues that were modified was studied by tryptic digestion in combination with LC-MS/MS analysis. As expected, the obtained fragments revealed that modification of Tyr was preferred over the modification of Trp. Singly modified lysozyme occurred on either Tyr23 or Tyr20 (Figure 2A, Table 1), which are the most exposed residues with SAA of $32 \%$ and $28 \%$, respectively. That site-specificity is not merely dictated by the amount of modification is apparent from the results obtained for G8 and PW17, which show very similar modification ability. Whereas the G8-based hGQ globally modifies lysozyme, the DNAzyme based on PW17 restricts its modification to one side of the protein (Figure 2A). Interestingly, when the PW17 sequence is conjugated to a lysozyme-binding aptamer (LBA), an additional modification of Tyr53 is observed (SI Table S6), which shows the potential influence of an aptamer on the modification ability of hGQ DNAzymes. ${ }^{30}$ Importantly, LBA itself was not able to enhance the background activity of hemin.

Following these encouraging results, we subjected human $\alpha$ thrombin $(33.6 \mathrm{kDa})^{31}$ to the same hGQDNAzyme sequences. Thrombin contains various Tyr and Trp residues that would be available for modification judging from their SAA (Figure 2B,C). As expected, hGQ DNAzymes displayed differences in their activity and site-specificity to modify thrombin, following comparable topology-related trends as was found for lysozyme (SI Tables S7-S9). For both lysozyme and thrombin, we found that cross-linking of the proteins did not occur in the absence of NML-derivative 1 (SI Figure S2).

LC-MS-MS analysis of tryptic digests of the reaction mixtures with thrombin revealed that in the presence of any of the hGQ DNAzymes, NML-derivative (1) and $\mathrm{H}_{2} \mathrm{O}_{2}$ modification took place on Tyr85 (SAA: 24\%) and Trp148 (SAA: 79\%) (Table 1, and SI Table S10). Depending on the G-quadruplex structure, additional modifications were detected (Figure 2B). Interestingly, two of the more exposed Tyr residues were not modified: Tyr71 (SAA: 62\%) and Tyr47 (SAA: 28\%) (Figure 2C). Tyr71 is located at the anion-binding exosite $\mathrm{I}$ of thrombin, which is also the known binding site of the G-quadruplex thrombin binding aptamer (TBA). ${ }^{32}$ Similarly, Tyr47 is located at the periphery of cationic exosite II, which is the binding site for thrombin binding aptamer HD22. Based on this, we propose that the hGQ DNAzymes interact at those sites, thereby blocking modification of these specific exposed residues.

Apparently, modification can be limited to only a few exposed residues. To examine if this also applied to larger proteins that potentially contain many more exposed reactive residues, we investigated modification of bovine serum albumin (BSA, 66 $\mathrm{kDa})$ and the monoclonal therapeutic antibody trastuzumab $(150 \mathrm{kDa})$. For these proteins, we used SDS-PAGE analysis and visualized the modification using a two-step labeling approach in which NML- $\mathrm{N}_{3}$-modified protein was derivatized by means of a strain-promoted alkyne-azide cycloaddition (SPAAC) reaction to a $4 \mathrm{kDa} \mathrm{BCN}$-functionalized PEG unit as mass-tag. As expected, both proteins were modified in the presence of NML$\mathrm{N}_{3}$ (1), $\mathrm{H}_{2} \mathrm{O}_{2}$, and hGQ DNAzymes (see SI Figures S3-S4). DNAzyme activities appear to be similarly related to the different topologies as was the case for lysozyme and thrombin. Specifically, whereas BSA was modified once by hemin alone, ${ }^{23}$ in the presence of GQ sequences, higher numbers of modifications were observed. The therapeutically relevant antibody trastuzumab was primarily modified on the heavy chain with up to three modifications for the most active hGQ DNAzymes (i.e., PW17 and cMyc) and higher concentrations of reagents (see SI Figure S5; modifications with the mass-tag appear more abundant on the $50 \mathrm{kDa}$ heavy chain than on the 25 $\mathrm{kDa}$ light chain). As observed for thrombin, the number of modifications decreased when the amount of DNAzyme was reduced 2- or 4-fold (i.e., from 0.9 equiv with respect to the protein, to 0.45 and 0.225 ). In addition, conditions might require optimization for each protein.

Now that we established that our protein modifying catalysts display features usually associated with enzymes (i.e., high rate, chemoselectivity, and site-specificity), we designed a system that allowed regulation of the hGQ catalyzed protein modification reaction by means of a switchable element (Figure 3A). ${ }^{33,34}$ Upon addition of an ssDNA sequence that is complementary to the PW17 sequence (i.e., an OFF switch), a DNA duplex would be formed that does not have the ability to activate hemin. Indeed, we were able to switch the activity of the DNAzyme between its active ("ON") and inactive ("OFF") forms by means of an external stimulus. Specifically, in the ON state, the DNAzyme modifies approximately $80 \%$ of lysozyme with NML derivative 1 , whereas in the OFF state, the modification conversion drops to $\sim 5 \%$, which is similar to hemin alone (see HPLC traces in the SI Figures S6-S11). Importantly, the hGQDNAzyme was formed again after addition of an activating ssDNA strand that was complementary to the ssDNA OFF strand. Since the activating strand contains a high number of guanine bases, we designed a strand that in itself does not form an active hGQ DNAzyme (see SI Figure S11). Indeed, the reformed hGQDNAzyme complex regained its original protein modifying ability, also when a DNAzyme-aptamer conjugate was applied (SI Figures S6-S9). Using the larger and fluorescent lissamine-NML conjugate $\mathbf{2}$, the switchable 


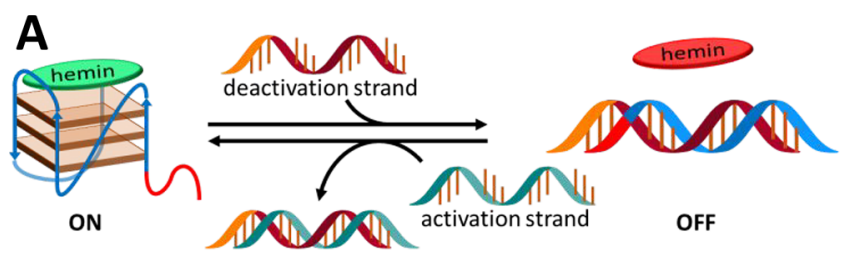

B
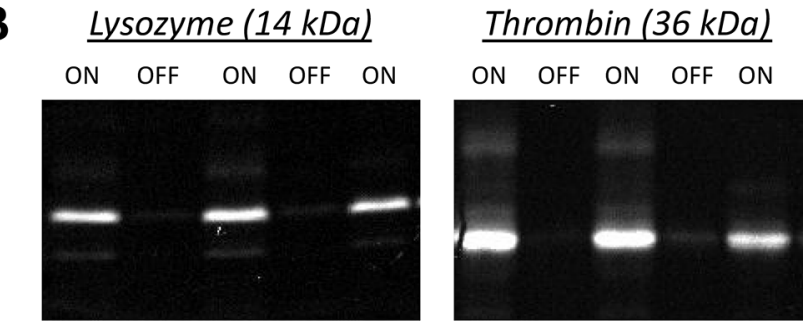

C

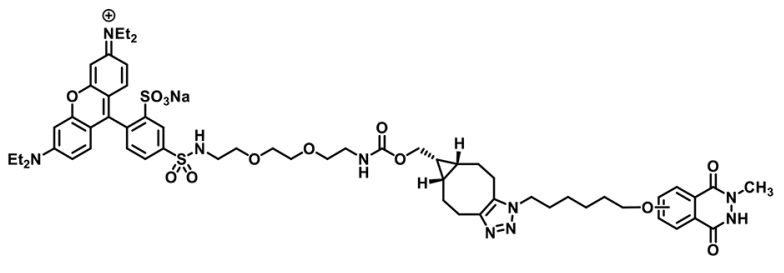

lissamine-NML, 2

Figure 3. (A) Design of the switchable hGQ DNAzyme system. (B) SDS-PAGE analysis of the trigger-regulated modification of lysozyme (left) and thrombin (right) by means of a fluorescent $\mathrm{N}$-methyl luminol derivative 2. (C) Structure of lissamine-NML conjugate 2.

character of the hGQDNAzyme was visualized by SDS-PAGE analysis for both lysozyme and thrombin (Figure 3B,C). We note that with derivative 2 the modification efficiency was reduced; with the smaller derivative (1), conversions of $80 \%$ were achieved.

In conclusion, we describe how the hemin/G-quadruplex (hGQ) DNAzyme nanostructure can be used for the oxidative modification of Tyr residues, and to a lesser extent Trp residues, with $\mathrm{N}$-methyl luminol derivatives in the presence of $\mathrm{H}_{2} \mathrm{O}_{2}$. In the absence of NML-tag, protein-protein cross-linking was not observed. We observed a correlation of the differences in protein modification and the GQ folding conformation, where the parallel GQ sequences are more active than antiparallel GQs. Furthermore, we found preferences for specific residues that are modified by the different GQ topologies. This suggests differences in interaction between the various hGQDNAzymes with the different target proteins, a process that can further be affected by the application of protein-binding aptamers. Lastly, we show that the catalytic nanostructure can be inactivated by the application of an external trigger, thereby lowering protein modification to the background activity of $<5 \%$ that we observed for hemin alone. The observed hGQ DNAzyme-catalyzed modification of proteins is novel, adding a yet unknown $\mathrm{C}-\mathrm{N}$ bond-forming reaction to the hGQ DNAzyme catalytic repertoire. Furthermore, the rapid rate of modification, its chemoselectivity, site-specificity (which is potentially influenced by the presence of a protein-binding aptamer), and ability to respond to an external trigger, make this biomimetic protein modification process not that dissimilar from biological enzymatic processes. In view of the many GQ structures that can bind to proteins, ${ }^{35}$ the growing applications for DNA nanotechnology ${ }^{36,37}$ and the importance of modified proteins for many lines of research in many scientific disciplines, we expect that our approach will uncover novel catalysts with specific protein modification abilities. Lastly, this study shows that DNA-based catalysts have the remarkable ability to modify proteins, and it is expected that DNA represents not only a genetic (indirect) entry to protein modification, but also direct access to modified proteins by means of their exquisite programmable catalytic functions. ${ }^{38}$

\section{ASSOCIATED CONTENT}

\section{(1) Supporting Information}

The Supporting Information is available free of charge at https://pubs.acs.org/doi/10.1021/acs.bioconjchem.0c00422.

Synthesis of the two NML derivatives, two full sets of conversion results for $22 \mathrm{hGQ}$ DNAzymes for lysozyme and thrombin (including HPLC traces of all reaction mixtures), additional experiments that determine the effect of variables, and SDS-PAGE gel images of BSA and trastuzumab (PDF)

\section{AUTHOR INFORMATION}

\section{Corresponding Author}

Bauke Albada - Laboratory of Organic Chemistry, Wageningen University \& Research, Wageningen 6807 WE, The Netherlands; ๑ orcid.org/0000-0003-3659-2434; Email: bauke.albada@ wur.nl

\section{Author}

Jordi F. Keijzer - Laboratory of Organic Chemistry, Wageningen University \& Research, Wageningen 6807 WE, The

Netherlands; $\odot$ orcid.org/0000-0001-7124-7500

Complete contact information is available at:

https://pubs.acs.org/10.1021/acs.bioconjchem.0c00422

\section{Author Contributions}

All authors have given approval to the final version of the manuscript.

\section{Funding}

Funded by the ECHO grant from the Dutch Organization for Scientific research (NWO) (project number 711.017.004).

\section{Notes}

The authors declare no competing financial interest.

\section{ACKNOWLEDGMENTS}

We thank Jorick Bruins for supplying $\mathrm{BCN}$-lissamine derivative that was used for lissamine-NML derivative 2, and Frank Claassen for his assistance on the LC-MS analysis. We thank Twan America for LC-MS/MS analysis on the tryptic digests of the modified proteins.

\section{ABBREVIATIONS}

NML, N-methyl luminol; LBA, lysozyme-binding aptamer; (h)GQ (hemin/)G-quadruplex; SAA, solvent accessible area.

\section{REFERENCES}

(1) Travascio, P., Li, Y., and Sen, D. (1998) DNA-enhanced peroxidase activity of a DNA aptamer-hemin complex. Chem. Biol. 5, 505-17.

(2) Stefan, L., and Monchaud, D. (2019) Applications of guanine quartets in nanotechnology and chemical biology. Nat. Rev. Chem. 3, 650-68. 
(3) Golub, E., Lu, C. H., and Willner, I. (2015) Metalloporphyrin/Gquadruplexes: from basic properties to practical applications. $J$. Porphyrins Phthalocyanines 19, 65-91.

(4) Li, W., Li, Y., Liu, Z., Lin, B., Yi, H., Xu, F., Nie, Z., and Yao, S. (2016) Insight into G-quadruplex-hemin DNAzyme/RNAzyme: adjacent adenine as the intramolecular species for remarkable enhancement of enzymatic activity. Nucleic Acids Res. 44, 7373-84.

(5) Cheng, X., Liu, X., Bing, T., Cao, Z., and Shangguan, D. (2009) General peroxidase activity of G-quadruplex-hemin complexes and its application in ligand screening. Biochemistry 48, 7817-23.

(6) Stefan, L., Denat, F., and Monchaud, D. (2012) Insights into how nucleotide supplements enhance the peroxidase-mimicking DNAzyme activity of the G-quadruplex/hemin system. Nucleic Acids Res. 40, 8759-72.

(7) Golub, E., Albada, B., Liao, W.-C., Biniuri, Y., and Willner, I. (2016) Nucleoapzymes: hemin/G-quadruplex DNAzyme-aptamer binding site conjugates with superior enzyme-like catalytic functions. J. Am. Chem. Soc. 138, 164-72.

(8) Albada, B., De Vries, J. W., Liu, Q., Golub, E., Klement, N., Herrmann, A., and Willner, I. (2016) Supramolecular micelle-based nucleoapzymes for the catalytic oxidation of dopamine to aminochrome. Chem. Commun. 52, 5561-64.

(9) Albada, B., Golub, E., and Willner, I. (2016) Rational design of supramolecular hemin/G-quadruplex-dopamine aptamer nucleoapzyme systems with superior catalytic performance. Chem. Sci. 7, 3092-101.

(10) Wang, F., Lu, C. H., and Willner, I. (2014) From cascaded catalytic nucleic acids to enzyme-DNA nanostructures: controlling reactivity, sensing, logic operations, and assembly of complex structures. Chem. Rev. 114, 2881-941.

(11) Hoyt, E. A., Cal, P. M. S. D., Oliveira, B. L., and Bernardes, G. J. L. (2019) Contemporary approaches to site-selective protein modification. Nat. Rev. Chem. 3, 147-71.

(12) Koniev, O., and Wagner, A. (2015) Developments and recent advancements in the field of endogenous amino acid selective bond forming reactions for bioconjugation. Chem. Soc. Rev. 44, 5495-551.

(13) Oliveira, B. L., Guo, Z., and Bernardes, G. J. L. (2017) Inverse electron demand Diels-Alder reactions in chemical biology. Chem. Soc. Rev. 46, 4895-950.

(14) Boutureira, O., and Bernardes, G. (2015) Advances in chemical protein modification. Chem. Rev. 115, 2174-95.

(15) Isenegger, P. G., and Davis, B. G. (2019) Concepts of catalysis in site-selective protein modifications. J. Am. Chem. Soc. 141, 8005-13.

(16) Sato, S., Nakamura, K., and Nakamura, H. (2017) Horseradishperoxidase-catalyzed tyrosine click reaction. ChemBioChem 18, 475-8.

(17) Zhang, Y., Park, K.-Y., Suazo, K. F., and Distefano, M. D. (2018) Recent progress in enzymatic protein labelling techniques and their applications. Chem. Soc. Rev. 47, 9016-136.

(18) Minamihata, K., Goto, M., and Kamiya, N. (2011) Site-specific protein cross-linking by peroxidase-catalyzed activation of a tyrosinecontaining peptide tag. Bioconjugate Chem. 22, 74-81.

(19) Bruins, J. J., Van de Wouw, C., Wagner, K., Bartels, L., Albada, B., and Van Delft, F. L. (2019) Highly efficient mono-functionalization of knob-in-hole antibodies with strain-promoted click chemistry. ACS Omega 4, 11801-7.

(20) Bruins, J. J., Blanco-Ania, D., Van der Doef, V., Van Delft, F. L., and Albada, B. (2018) Orthogonal, dual protein labelling by tandem cycloaddition of strained alkenes and alkynes to ortho-quinones and azides. Chem. Commun. 54, 7338-41.

(21) Bruins, J. J., Westphal, A. H., Albada, B., Wagner, K., Bartels, L., Spits, H., Van Berkel, W. J. H., and Van Delft, F. L. (2017) Inducible, site-specific protein labeling by tyrosine oxidation-strain-promotoed ( 4 +2 ) cycloaddition. Bioconjugate Chem. 28, 1189-93.

(22) Gilmore, J. M., Scheck, R. A., Esser-Kahn, A. P., Joshi, N. S., and Francis, M. B. (2006) N-terminal protein modification through biomimetic transamination reactions. Angew. Chem., Int. Ed. 45, 5307-11.

(23) Sato, S., and Nakamura, H. (2019) Protein chemical labeling using biomimetic radical chemistry. Molecules 24, 3980.
(24) Burge, S., Parkinson, G. N., Hazal, P., Todd, A. K., and Neidle, S. (2006) Quadruplex DNA: Sequence, topology and structure. Nucleic Acids Res. 34, 5402-15.

(25) Sato, S., Matsumura, M., Kadonosono, T., Abe, S., Ueno, T., Ueda, H., and Nakamura, H. (2020) Site-selective protein chemical modification of exposed tyrosine residues using tyrosine click reaction. Bioconjugate Chem. 31, 1417-24.

(26) Sato, S., Nakamura, K., and Nakamura, H. (2015) Tyrosinespecific chemical modification with in situ hemin-activated luminol derivatives. ACS Chem. Biol. 10, 2633-40.

(27) Esque, J., Oguey, C., and De Brevern, A. G. (2011) A novel evaluation of residue and protein volumes by means of Laguerre tessellation. J. Chem. Inf. Model. 51, 493-507.

(28) Li, W., Li, Y., Liu, Z., Lin, B., Yi, H., Xu, F., Nie, Z., and Yao, S. (2016) Insight into G-quadruplex-hemin DNAzyme/RNAzyme: adjacent adenine as the intramolecular species for remarkable enhancement of enzymatic activity. Nucleic Acids Res. 44, 7373-84.

(29) Shugar, D. (1952) The measurement of lysozyme activity and the ultra-violet inactivation of lysozyme. Biochim. Biophys. Acta 8, 302-9.

(30) Bastian, A. A., Marcozzi, A., and Herrmann, A. (2012) Selective transformations of complex molecules are enabled by aptameric protective groups. Nat. Chem. 4, 789-93.

(31) Villanueva, G. B., Perret, V., and Fenton, J. W., 2nd (1984) Conformational integrity of human $\alpha$-thrombin. Thromb. Res. 36, 37787.

(32) Pica, A., Russo Krauss, I., Parente, V., Tateishi-Karimata, H., Nagatoishi, S., Tsumoto, K., Sugimoto, N., and Sica, F. (2017) Through-bond effects in the ternary complexes of thrombin sandwiched by two DNA aptamers. Nucleic Acids Res. 45, 461-9.

(33) Wang, F., Liu, X., and Willner, I. (2015) DNA switches: from principles to applications. Angew. Chem., Int. Ed. 54, 1098-129.

(34) Liu, X., Niazov-Elkan, A., Wang, E., and Willner, I. (2013) Switching photonic and electrochemical functions of a DNAzyme by DNA machines. Nano Lett. 13, 219-25.

(35) Ray, S., Tillo, D., Boer, R. E., Assad, N., Barshai, M., Wu, G., Orenstein, Y., Yang, D., Schneekloth, J. S., and Vinson, C. (2020) Custom DNA microarrays reveal diverse binding preferences of proteins and small molecules to thousands of G-Quadruplexes. ACS Chem. Biol. 15, 925-35.

(36) Chidchob, P., and Sleiman, H. (2018) Recent advances in DNA nanotechnology. Curr. Opin. Chem. Biol. 46, 63-70.

(37) Bujold, K., Lacroix, A., and Sleiman, H. (2018) DNA nanostructures at the interface with biology. Chem. 4, 495-521.

(38) Silverman, S. K. (2015) Pursuing DNA catalysts for protein modifciation. Acc. Chem. Res. 48, 1369-79. 